\title{
What do the conditions of exact pseudospin symmetry in nuclear relativistic models mean in real nuclei?
}

\author{
B. Desplanques ${ }^{1}$ and S. Marcos ${ }^{2, a}$ \\ ${ }^{1}$ LPSC, Université Joseph Fourier Grenoble 1, CNRS/IN2P3, INPG, F-38026 Grenoble Cedex, France \\ ${ }^{2}$ Department of Modern Physics, University of Cantabria, E-39005 Santander, Spain
}

\begin{abstract}
We study the continuous evolution of the single-particle levels from a model satisfying exact spin symmetry (SS) to another one satisfying exact pseudospin symmetry (PSS), using a Dirac equation with potentials of scalar, vector and tensor type. All the standard pseudospin doublets (PSDs) appearing in the limit of the SS have their corresponding PSDs in the limit of the PSS but not vice versa. The PSS is strongly violated between the SS and PSS limits.
\end{abstract}

\section{Introduction}

In the last fifteen years, the pseudospin symmetry in atomic nuclei has been based by different authors [1-4] on the fact that, in the relativistic framework, the magnitude of the sum of the scalar $\left(\Sigma_{S}\right)$ and the time-like vector $\left(\Sigma_{0}\right)$ components of the nucleon self-energy is small, since the exact PSS is satisfied in the limit $\Sigma_{S}+\Sigma_{0}=0$. However, it has been argued in [5] that this result has only physical meaning for those systems that in the limit $\Sigma_{S}+\Sigma_{0}=0$ are bound. Consequently, since in this limit real nuclei are unbound, there is no reasons to ground the quasi-degenerate pseudospin doublets appearing in real nuclei on the fact that $\left|\Sigma_{S}+\Sigma_{0}\right|$ is small (see also [6-9]). Furthermore, in a nonrelativistic model [10], it was found that standard PSDs, appearing in conditions similar to those of real nuclei, do not correspond to the PSDs found in the limit of exact PSS. Actually, the true PSDs strongly violate the PSS in conditions of real nuclei. The aim of this work is to investigate whether such conclusions can also be stated in relativistic models of the atomic nuclei.

\section{Relativistic model enabling the transition between the SS and PSS limits}

The Dirac equation for a nucleon moving inside the nucleus subjected to the $\Sigma_{S}$ and $\Sigma_{0}$ potentials as well as a potential i $\beta \boldsymbol{\alpha} \cdot \hat{\mathbf{r}} U(r)$ of tensor character can be written in standard form as [11]

$$
\left[\boldsymbol{\alpha} \cdot \mathbf{p}+\beta M+\beta \Sigma_{S}(r)+\Sigma_{0}(r)+i \beta \boldsymbol{\alpha} \cdot \hat{\mathbf{r}} \Sigma_{T}(r)\right] \psi(\mathbf{r})=E \psi(\mathbf{r})
$$

where $\psi(\mathbf{r})$ represents the nucleon Dirac spinor.

\footnotetext{
ae-mail: marcoss@unican.es
} 
In order to study the transition between two models A and B satisfying exact SS and PSS, respectively, we consider the potentials $V(r)=\Sigma_{0}(r)+\Sigma_{S}(r)$ and $\Delta(r)=\Sigma_{0}(r)-\Sigma_{S}(r)$ entering the Dirac equation (1) as

$$
\begin{gathered}
V(r)=(1-x) \frac{8 M}{1+e^{-\left(r-r_{0}\right) / a}}\left[\frac{1}{1+e^{\left(r-r_{0}\right) / a}}+\eta \frac{1}{1+e^{-\left(r-r_{0}\right) / a}}\right], \\
\Delta(r)=x \frac{4 M}{1+e^{-\left(r-r_{0}\right) / a}},
\end{gathered}
$$

where, we take, somewhat arbitrarily, $a=0.1, M=10 \mathrm{fm}^{-1}$ (as in [11-13]), $r_{0}=2 \mathrm{fm}$, and

$$
\eta=\frac{4\left(x_{0}-x\right)}{1+100\left(x-x_{0}\right)^{2}}
$$

with

$$
x_{0}=\frac{E+M}{4 M} .
$$

Thus, if $\Sigma_{T}(r)=0$, for $x=0$ we recover the model A, which satisfies exact SS, whereas for $x=1$, we have the model B, which satisfies exact PSS. We do not expect our main conclusions will depend very much on our specific choice of parameters. As in [11], the potentials $V(r)$ at $x=0$ and $\Delta(r)$ at $x=1$ take positive values and are rather flat in the vicinity of $r=0$, whereas in the large- $r$ limit, they are bounded by an upper finite value instead to increase infinitely.

To facilitate the understanding of the solutions of the Dirac equation (1) with these potentials, it is useful to consider the corresponding equivalent Schrödinger equation for the large component (see [5] for details). In this equation, with the potential $\Delta(r)$ that becomes constant for $r \rightarrow \infty$, the central potential, $U(r)$, at $r \rightarrow \infty$ can be approximated as

$$
U(r \rightarrow \infty)=V+\frac{1}{2 M}\left[e(V+\Delta)-e^{2}-V \Delta\right],
$$

where $e \equiv E-M$. Notice that for $V$ small enough, $U(r \rightarrow \infty)$ becomes essentially energy dependent.

The last term in Eq. (2), $\propto \eta$, makes $V(r \rightarrow \infty)$ different from zero. Its sign changes at $x=x_{0}$ to make the central potential $U(r)$ attractive at large values of $r$. The factor 4 entering the expression for $\eta$ in Eq. (4) controls the "speed" of the transition from the SS to the PSS limit as $x$ varies. The factors 100 and 4 determine the value of $V(r)$ at $r \rightarrow \infty$ for a given value of $x$. It is easy to see that $U(r \rightarrow \infty)=e$ for $x=x_{0}$. Thus, for $x \rightarrow x_{0}$, the system spreads all over the space. To avoid this problem, we consider $\Sigma_{T}(r) \neq 0$. We choose

$$
\Sigma_{T}(r)=\frac{10}{1+e^{-\left(r-r_{0}\right) / a}} \times e^{-\frac{1}{2}\left[\left(x-x_{0}\right) / a\right]^{2}},
$$

which takes relevant (though small) values only around $x \approx x_{0}$.

\section{Results}

With the potentials given in Sect. 2, the results of the Dirac equation (1) for the $s p$ energies of a number of states are drawn in Fig. 1. For the states appearing at $x=0$, the $s p$ energies vary continuously from $x=0$ (SS limit) to $x=1$ (PSS limit), although the number of nodes (n) of the large component of their Dirac spinor increases by one unit for $x \gtrsim x_{0}$. Thus, for states that only appear in this region and for states in the PSS limit we use the notation $(n-1) l_{j}$ instead of $n l_{j}$. In most of cases, the $s p$ energies vary with $x$ smoothly around $x \approx x_{0}, x_{0}$ taking values close to 0.5 . Apart from 
the special (relativistic) state $0 f_{5 / 2}$, only the states $1 f_{5 / 2}$ and mainly the $1 g_{7 / 2}$ (with $n=1, l$ large and $j=l-1 / 2$ ) show $s p$ energies that vary quite sharply around $x \approx x_{0}$. By including a large density of points in Fig.1, the three narrow picks for the $s p$ energy become somewhat more even and their height decreases as the strength of $\Sigma_{T}(r)$ potential increases. However, the strong increase of the $s p$ energy shown for all states with $j=l-1 / 2$ for $x \gtrsim 0.6$ is almost not affected by $\Sigma_{T}(r)$. This behaviour is due to the strong modifications of the two components of the Dirac spinor as $x$ varies.

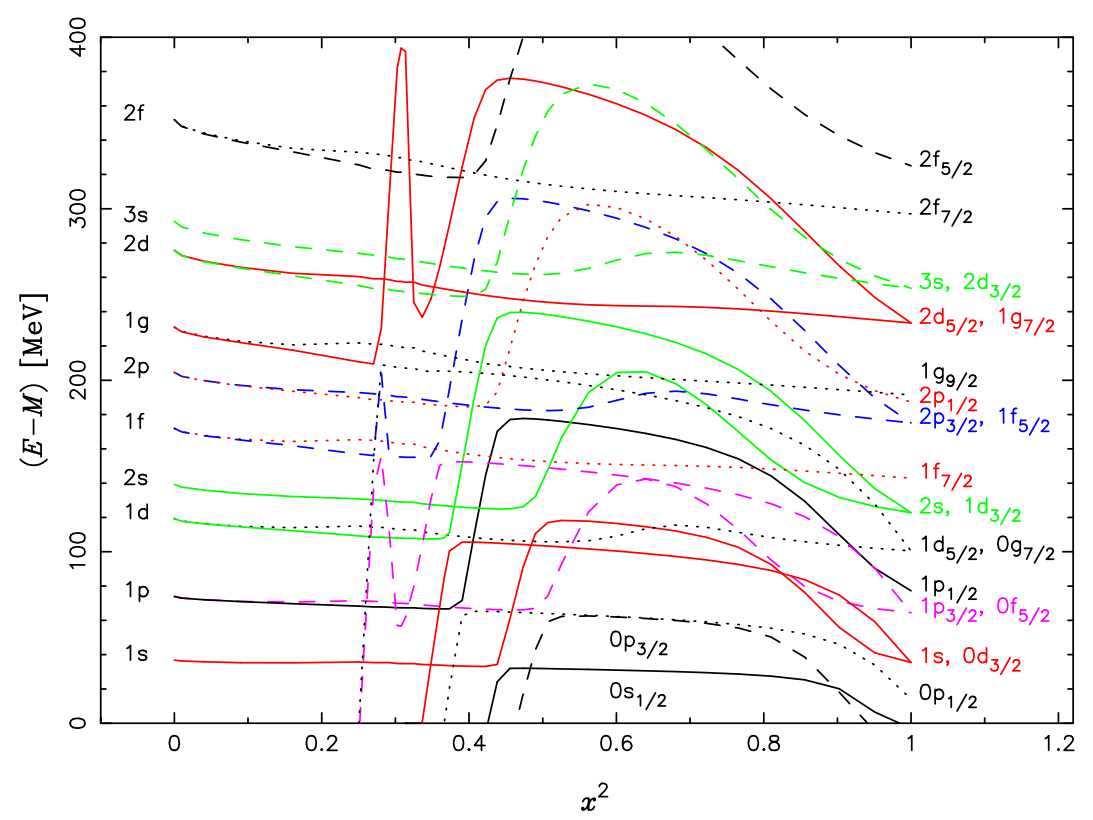

Figure 1. Evolution of the $s p$ levels as a function of $x$ from $x=0$ (SS limit) to $x=1$ (PSS limit). Our conventions for the potentials at $x=0$ imply positive values for $E-M$ in this limit.

For the states with $j=l+1 / 2$, the extra nodes of the big component appearing for $x \gtrsim x_{0}$ are located in a domain where the contribution to the norm beyond this node is, relatively, small (this observation is similar to one we could make for the non-relativistic models considered in [10] or for the relativistic ones given in [11-13]. As a counterpart to the apparition of extra nodes, all the states with the only node at $r \rightarrow \infty$ in the PSS limit (i.e., the PS partner of the intruder states) cannot be related to any state in the SS limit. As seen from the figure, they acquire a negative energy and "disappear" from the positive-energy spectrum for $x$ close to $x_{0}$. This fact explains the absence of the PS partners of the intruder states in real nuclei. It is also seen from the figure that other states with the only node at $r \rightarrow \infty$, different from the PSS intruder partners, appear in the region $\left[x_{0}<x<1\right]$, without spoiling the symmetry properties of the spectra obtained in the PSS limit.

The appearance of the states noted as $0 l_{j}$ for $x>x_{0}$ strongly influences the spectra of the states noted as $1 l_{j}$ that have bigger energy (and these states influence the $2 l_{j}$ states, etc.), "pushing" them upwards (when their energies are very close to each other), evidencing very strongly level-repulsion properties for $x$ in the interval $\left[x_{0}<x<0.7\right]$. In this range, the wave functions can be strongly changed, $n \rightarrow n+1$ and their variations with $x$ can be highly non perturbative ${ }^{1}$. In some cases,

\footnotetext{
${ }^{1}$ The non perturbative character of the PSS, which is nowadays generally accepted, was first shown in [14] and studied in detail in [15]. Notice, however, that, for $V(r) \propto r^{2}$ and $\Delta(r)=0$, one gets not very unrealistic nuclei and degenerate PSDs, although the PSS (as derived from the condition $V(r)=0$ [1-3]) is very strongly broken [5, 7, 8, 11-15].
} 
the "small" component of the Dirac spinor could become larger than the "big" one, suggesting the essential role of relativity in this process.

The details of the above results depend on the transition model, but the appearance of an extra node as well as the appearance (or disappearance) of some states from the positive part of the $s p$ spectrum when going from the SS limit to the PSS one can be considered as genuine features that point to a drastic breaking of the PSS when $x$ decreases from $x=1$ to $x=0$, approaching real nuclei.

\section{Conclusions}

In this paper, we have examined whether there could be a continuous transition for the $s p$ levels of the atomic nuclei between the SS and the PSS limits and have analyzed what would be the implications of such a result. We have used a relativistic approach, as this one has been thought to provide some support for the existence of an approximate PSS in real nuclei. However, while all states in the SS limit can be continuously related to states in the PSS limit, the opposite is not true. The PS partners of the so called intruder states in the PSS limit disappear from the positive-energy spectrum near $x \approx x_{0}$ as $x$ decreases and cannot be related to any state in the SS limit, throwing light about the reasons of absence of those PS partners in the SS limit (and in configurations of real nuclei).

This result together with the strong splitting of the PSDs and strong modifications of the two components of the Dirac spinors for $x_{0}<x<1$ point to a very strong violation of the PSS, which, in this relativistic case, can be considered more important than the one found in [10], using a nonrelativistic approximation, where only a re-ordering of the $s p$ levels was observed. The transition from the PSS limit to the SS limit, close to real nuclei, is very strongly not perturbative.

From examining wave functions, it was shown in the literature $[5,6,8]$ that the PSS (as defined in [1-4] and in the present work) seems to be not as good as the SS, pointing out to the accidental character of the quasi-degenerate PSDs appearing in real nuclei. Starting from a different point of view, the results of the present work strongly support this conclusion.

\section{Acknowledgments}

This work has been supported by the MEC grant FIS2005-04033.

\section{References}

[1] J.N. Ginocchio, Phys. Rev. Lett. 78, 436 (1997);

[2] J.N. Ginocchio, D.G. Madland, Phys. Rev. C 57, 1167 (1998);

[3] J.N. Ginocchio, Phys. Rep. 414, 165 (2005); Nucl. Phys. News 15, 13 (2005);

[4] J. Meng, K. Sugawara-Tanabe, S. Yamaji, P. Ring, A. Arima, Phys. Rev. C 58, R628 (1998);

[5] S. Marcos, M. López-Quelle, R. Niembro, L.N. Savushkin, Eur. Phys. J. A 37, 251 (2008);

[6] S. Marcos, M. López-Quelle, R. Niembro, L.N. Savushkin, Eur. Phys. J. A 20, 443 (2004);

[7] S. Marcos, M. López-Quelle, R. Niembro, L.N. Savushkin, J. Phys G: Nucl. Part. Phys. 31, S1551 (2005);

[8] S. Marcos, V.N. Fomenko, M. López-Quelle, R. Niembro, L.N. Savushkin, Eur. Phys. J. A 26, 253 (2005);

[9] S. Marcos, M. López-Quelle, R. Niembro, L.N. Savushkin, Eur. Phys. J. A 34, 429 (2007);

[10] B. Desplanques, S. Marcos, Eur. Phys. J. A 43, 369 (2010);

[11] R. Lisboa, M. Malheiro, A.S. de Castro, P. Alberto, M. Fiolhais, Phys. Rev. C 69, 024319 (2004);

[12] T. S. Chen et al., nucl-th/0205021;

[13] T.S. Chen, H.F. Lü, J. Meng, S.O. Zhang, and S.G. Zhou, Chin. Phys. Lett. 20, 358 (2003).

[14] S. Marcos, M. López-Quelle, R. Niembro, L. N. Savushkin, and P. Bernardos, Phys. Lett. B 513, 30 (2001).

[15] H. Liang, P. Zhao, Y. Zhang, J. Meng and N. Van Giai, Phys. Rev. C 83, 041301(R) (2011). 